\title{
Naive Infinitism: The Case for an Inconsistency Approach to Infinite Collections
}

\author{
Toby Meadows
}

\begin{abstract}
This paper expands upon a way in which we might rationally doubt that there are multiple sizes of infinity. The argument draws its inspiration from recent work in the philosophy of truth and philosophy of set theory. More specifically, elements of contextualist theories of truth and multiverse accounts of set theory are brought together in an effort to make sense of Cantor's troubling theorem. The resultant theory provides an alternative philosophical perspective on the transfinite, but has limited impact on everyday mathematical practice.
\end{abstract}

This relativity of cardinalities is very striking evidence of how far abstract formalistic set theory is removed from all that is intuitive. One can indeed construct systems that faithfully represent set theory down to the last detail. But as soon as one applies the finer instruments of investigation all this fades away to nothing. Of all the cardinalities only the finite ones and the denumerable one remain. Only these have real meaning; every thing else is formalistic fiction.

—John von Neumann [33, p. 408]

The aim of this paper is to take seriously the idea that we have, in some sense, misunderstood the message of Cantor's theorem, or, at the least, that in hindsight we have driven headlong into the transfinite when we could have paused a moment longer to consider an alternative. My goal is to demonstrate that Cantor's theorem can be understood more like the liar paradox, as a kind of fork in the road. The crucial idea is that, in admitting that there are multiple sizes of infinity, we have done irreparable

Received May 9, 2012; accepted December 9, 2013

2010 Mathematics Subject Classification: Primary 03A05, Y002; Secondary 03E99

Keywords: Cantor's theorem, set theory, the liar paradox, truth, Tarski, Kripkean truth, forcing, generic elements

(C) 2015 by University of Notre Dame 10.1215/00294527-2835074 
damage to our naive conception of the infinite. My goal in this paper is to demonstrate that we may coherently reject the multiplicity of infinite cardinalities and to illustrate the value of this perspective. ${ }^{1}$

Now there is, of course, an extensive, infamous, and largely invisible body of literature consisting of attempts to refute Cantor's theorem (see Hodges [17]). This paper is not intended as a contribution to that corpus. My contention is that, actually, it is not so difficult to make intelligible something like the view suggested above. The difficulty, rather, lies in showing that it is interesting.

The paper is broken into three main sections: the Setup; the Case of the Missing Bijection; and Problems. The first section dominates the paper. The goal here is to lay out the problem as clearly as possible. We articulate the problem in two passes. The first pass formulates a version of Cantor's theorem from a pre-set-theoretic framework, after which we initiate discussion as to its interpretation. We then introduce and formulate an analogy between Cantor's theorem and the liar paradox. In the second pass, we take up a version of third-order arithmetic and from there argue that like the liar paradox, we may consider Cantor's theorem as presenting us with a fork in the philosophical road. In the second section, we discuss a problem opened in the first section. We sketch a response to it which sheds some light on a possible link between contemporary contextualist accounts of truth and multiverse approaches to set theory. Finally, we address some of obvious problems for the view proposed.

\section{The Setup}

Let us suppose, for the sake of this argument, that we have no quibbles with the structure of the natural numbers: that quantification over the natural numbers presents no problems with regard to intelligibility or ambiguity. Then suppose it becomes convenient for us to talk about collections of natural numbers. Perhaps we have need for analytic number theory, or maybe we want to prove the completeness of a logic coded by integers. Proceeding in this naive fashion, the following question should eventually leap out at us.

Question 1.1 What tells us that the natural numbers themselves cannot be used to represent these classes?

Of course, the obvious answer-which we learned long ago-is that there are just too many classes for us to do this. Cantor's theorem tells us that there is no bijection between the naturals and the classes of naturals, so no such representation can work. Surely this is the right answer, but this delivery of the argument is too swift. A couple of important ancillary questions immediately crop up:

(1) What theoretical background must we already have accepted to find the theorem compelling?

(2) What is the nature of our compulsion to accept the conclusion of the argument in Cantor's theorem?

In addressing the second question, it may be useful to draw a rough distinction between philosophical and mathematical compulsion. I am thinking here of a certain difference between philosophical and mathematical arguments and their normative effect. By a mathematical argument, I have in mind a proof constructed from axioms using logical rules, the result of which is a theorem. Rejecting the conclusion of such an argument is usually very difficult as it tends to require revision of deep 
beliefs. Nonetheless, one may reject one of the axioms, leaving us with a valid although unsound argument. On the other hand, a philosophical argument-while it may be mathematical —also has recourse to a wider variety of persuasive machinery. For example, pragmatic considerations such as simplicity and elegance may come into play when arguing for a particular conclusion. Rejecting the conclusion of an argument which relies on extramathematical techniques is usually easier as both the premises and the moves made within it are more viable for revision.

I suspect that the answer to the first question is that many of us tend to think of arithmetic as being adequately represented in set theory, and such a theory is essentially correct. From this perspective Cantor's theorem is easy to both formulate and prove. Moreover in answer to the second question, from this point of view, our compulsion to accept the conclusion has mathematical force: it is a theorem. However, there seems to me a sense in which giving ourselves the full power of set theory begs the question. Underlying this investigation is a question about what motivates us to accept such a theory of sets. These answers merely tell us what we should do when we already accept such a theory. I would like to take a slight step back from this precipice. Intuitively speaking, I want us to imagine a fictitious moment in history before the advent of set theory but in which we possess a full toolkit for logical inquiry.

1.1 A first pass Our goal in this section is to formulate and prove Cantor's theorem from a modest pre-set-theoretic perspective. This should illustrate how Cantor's theorem could be used to show that the natural numbers are not sufficient for the representation of classes. We shall then discuss what we ought to make of the result. Given our assumption of comfort around arithmetic, we shall take a theory of arithmetic as our starting point and from there develop a position from which Cantor's theorem can be articulated. Our first problem is that we need to be able to refer to classes and we have no obvious representatives in our language.

Problem 1.1 We want to talk about classes of natural numbers.

To deal with this, let us introduce uppercase variables $X, Y, \ldots$ to represent classes in our theory. This is, of course, going to result in something which looks like secondorder arithmetic as found in, say, Simpson [30]. However, our use of classes here is intended to be purely instrumental. We are not presupposing anything about them. Indeed, we are trying to ascertain whether they are superfluous.

Problem 1.2 Can we use natural numbers to represent classes?

We want to leave open the possibility that the numbers themselves could do the work of classes, but we also need to leave open the possibility that they might not. We do not want to make any assumptions either way at this point. However, if we could use natural numbers for this purpose, then there would need to be a bijection between the natural numbers and the classes of natural numbers.

The next problem is the statement of Cantor's theorem. Usually, we state it by saying that there is no bijection from the naturals to the powerset of them. However, prima facie, this statement involves a third-order function. Given that our current stance makes us a little hesitant around second-order objects, we would be better served leaving third-order objects off the table, at least for the moment. Thus, we shall first articulate the statement of the theorem in terms of classes. We assume that 
$\langle\cdot, \cdot\rangle: \omega \times \omega \rightarrow \omega$ is a bijection. With this in hand, we may then state a version of the theorem as follows.

\section{Theorem 1.1 $\neg \exists Y \forall X \exists ! n \forall m(m \in X \leftrightarrow\langle n, m\rangle \in Y)$.}

Intuitively, this says that there is no $Y$ which codes a bijection between natural numbers and the classes thereof. To prove this, we shall assume that some kind of comprehension principle (Comp) is acceptable. Assuming that the bijection is a recursive function, then we only need recursive comprehension (with parameters) to formulate the argument. In the reverse mathematics literature, such a theory is known as $R C A_{0}$ and is the weakest comprehension principle in general use (see [30]). Intuitively speaking, it allows us to prove the existence of all the Turing machines. While we could formulate weaker comprehension axioms, which would block the argument, they would be arguably too weak for any interesting mathematical purposes. As such, such weakness may tell against our initial motivations regarding the congeniality of quantifying over classes of natural numbers. With this in mind, we shall not attempt to describe the correct comprehension axiom here, but we shall demand that we can at least prove that given any set, we may prove the existence of any set which is recursive in it. $^{2}$

Proof Suppose that there were such a $Y$, then by (Comp), there will be some $B$ such that

$$
\forall m(m \in B \leftrightarrow\langle m, m\rangle \notin Y) .
$$

By assumption there is some $b$ such that

$$
\forall m(m \in B \leftrightarrow\langle b, m\rangle \in Y) .
$$

Now suppose $b \in B$. Then $\langle b, b\rangle \in Y$ and thus $b \notin B$. But suppose $b \notin B$. Then $\langle b, b\rangle \in Y$ and $b \in B$. This is a contradiction. Thus there is no such $Y$.

1.1.1 Interpreting the result This is very familiar, but let us now take some time to consider how the result should be interpreted. At face value, it says that there is no class witnessing a bijection between the natural numbers and the classes of natural numbers. But what does it mean for a theory to say this? There appear to be a couple of ways we could understand this:

(1) we could see it as saying that there is no such bijection; or

(2) we could see it as saying that our theory is incapable of procuring such a bijection.

They do not necessarily mean the same thing. Clearly, something like (1) is the traditional interpretation. The theorem states that there is no bijection and so we say that there is none.

The second approach, and the approach outlined in this paper, is not traditional and is likely to appear subtle or sophisticated. First, we should note that just because some theory refutes the existence of an object, this does not always mean that we should reject the existence of it. For example, the theory could turn out to be false or inconsistent. Along these lines, my contention is that there is another way of interpreting the theorem. Rather than seeing the result as a proof of the nonexistence of a bijection, we might see it as a failure of our theory to quantify over all the subsets of the naturals. Indeed, we might see it as a necessary failure to do so. The upshot is not that the bijection fails to exist, but rather that our theory has failed to quantify 
over everything we intended it to. For one thing, we would say that it missed the class which coded the bijection. This leads us to a difficult question.

Problem 1.3 How do we make sense of the missing bijection?

What can it mean for a theory to necessarily fail to quantify over the objects we intend it to? First, we might think of this as speaking of some kind of epistemic limitation or inability for us to express something which we intend to say. However, I think that this way of interpreting matters cuts deeper than that. I am interested in entertaining the idea that the range of the quantification in this theory is actually incoherent. The key and contentious idea is that there is no powerset of the naturals to quantify over. There is no theory than can quantify over all the subsets of the natural numbers. We shall return to this problem in the next section of the paper.

This a bare outline of the position. Unless one is of an intuitionistic persuasion, it should, at present, appear to stand four-square against all that is orthodox and sensible in the philosophy of mathematics. ${ }^{3}$ As such, this would give us good reason to think that it is uninteresting, misguided, or outright incoherent. My goal for the rest of the paper is to defend the rationality of the upholder of this view. I shall do this in two parts. First, we shall exploit an analogy with truth to provide a more precise formalization of the view proposed. The desired upshot here is a demonstration of the coherence of the viewpoint. Second, we shall be more pragmatic and investigate the costs and benefits associated with this view. Our goal here is to show that there is no ultimately persuasive reason to think the position is false.

1.2 An analogy with truth On the road to making sense of the above discussion, we now explore an analogy between Cantor's theorem and Tarski's theorem [31], [32] regarding the inadmissibility of truth. Usually, Tarski's theorem is known as a theorem about the undefinability of truth. Thus, we usually suppose that we are using theory with sufficient expressive resources to talk about its own syntax, and we suppose that we have used this language to construct a formula $\psi(x)$ with one free variable such that for all sentences $\varphi$ of the language we can show in our theory that $\varphi$ if and only if $\psi\ulcorner\varphi\urcorner{ }^{4}$ Then using the diagonal lemma, which will be present in a theory with this expressive power, we construct a sentence $\lambda$ such that $\lambda$ if and only if $\neg \psi\ulcorner\lambda\urcorner$. From here, it is a simple matter to demonstrate that the resultant theory is inconsistent. The assumption that we could define such a formula was therefore incorrect.

There is a sense in which the inadmissibility reading is stronger. We do not worry about whether such a formula can be defined; we merely ask whether we could expand a language with a new predicate $T$ such that for all sentences $\varphi$ of the expanded language it is the case that $\varphi$ if and only if $T\ulcorner\varphi\urcorner$. To achieve this we simply expand the theory with a new axiom $\varphi \leftrightarrow T\ulcorner\varphi\urcorner$ for every sentence $\varphi$. We call these $T$-axioms. Once again, we exploit the diagonal lemma and show that such a theory is inconsistent; thus there is no way of expanding the language with a predicate that enjoys the property described above. For this reason we say that truth is inadmissible.

Now, if we squint a little, there does seem to be a similarity between Tarski's theorem and Cantor's. Both proofs make use of some kind of self-referring diagonal technique and then exploit this to show that a prima facie intuitive assumption cannot hold. In Tarski's theorem, we show that is not the case that a truth predicate can be admitted. In Cantor's theorem, we show that it is not the case that a bijection exists 
between the naturals and the sets of naturals. But is this merely cosmetic? My goal is to show that it is not.

I intend to expand upon an alternative interpretation of Cantor's theorem, although I would like to stress that by no means am I trying to refute it. From a wide-angle view, I would like to show that Cantor's theorem should be treated more as a fork in the road than as a surprising result which nonetheless pushes us to theorize subsequently in a particular way. In contrast to the prevailing view, I am aiming to demonstrate that Cantor's theorem could show us that we were mistaken to think we could unproblematically quantify over all of the sets of natural numbers. Moreover, I would like to suggest that there is a relationship here with well-known problems regarding the truth predicate. To see this, we observe that a common philosophical diagnosis of the inadmissibility of the truth predicate is to assert that we were mistaken to think we could ever genuinely quantify over all of the sentences in a semantically closed language. We now attempt to draw out this analogy.

To get things moving, I make the following intuitive contention.

\section{Claim 1.2 The idea of there being multiple sizes of infinity is very strange and} mysterious.

I do not propose to argue for this claim-I doubt that it is susceptible to a satisfying philosophical defense. I shall, however, assume that the reader has, at least at some point, shared this intuition-presumably before their first encounter with the theorem.

We do, however, observe that the idea of infinity being strange is not without historical precedent. Before Cantor came along, infinity-if it was countenanced at all-was a size larger than any size - arguably not even a size at all. In measuring the size of infinite collections of natural numbers, Paolo Mancosu [23, p. 613] provides a rich historical discussion of philosophical attitudes to infinite collections. In particular, he attributes the following paradox to Galileo:

(1) There are more natural numbers than squares.

(2) The collection of natural numbers has as many elements as the collection of squares.

Intuitively, we are pulled toward (1) since so many natural numbers are not square numbers at all. On the other hand, we are pulled toward (2) since, for every number, we can find a square number corresponding to it. This kind of confusion is deeply pre-Cantorian and not the focus of this paper. Nonetheless, it is reflective of the difficulties philosophers and mathematicians faced in reconciling the infinite to naive conceptions of size. ${ }^{5}$

Our position in this paper does not fit easily in this schema. For extrinsic reasons, we have agreed from the outset to countenance talk of infinite collections, but in the next section we shall see how this leads to paradox. Depending on how matters are made precise, we sit somewhere around (1) and (2).

We shall take it that to talk of multiple sizes of infinity is simply absurd. Moreover, this intuition forms the basis of our argument. In looking for something to blame, we shall look to our theory of infinite collections and argue that something has gone awry. 
1.3 The second pass To draw out our analogy, we are now going to formalize an admissibility argument regarding theories of infinite collections. To do this, we need some way of articulating our assertion that

(Inf) there is only one size of infinity.

This will play an analogous role as the $T$-axioms in the theory of truth. From here, we show that this leads to inconsistency and thus that we are obliged to change something in our theory. We first observe a way that does not work. It will not do to stay in our theory of classes of naturals and state that every class $X$ is such that either

- $X$ is finite; or

- there is a bijection between $X$ and the naturals.

While we can articulate this in the language of classes and numbers described above, it cannot work for our purpose since there are never going to be any classes of naturals which are larger than the class of all naturals. So to draw out the analogy, we need to get into a position where we can articulate the problem. According to the traditional reading, Cantor's theorem tells us that the collection of all classes of naturals is larger than any class of naturals. Thus we need to be able to talk about collections of classes as well as classes. To do this, we shall move into something like third-order arithmetic. We shall introduce a third level of objects which can refer to what we shall call families of classes. Once again, we are making no commitments as to what families are or whether or not they too could be represented by naturals or classes of naturals.

We augment our formal language with a collection of family variables which we represent with uppercase calligraphic letters: $\mathcal{X}, \mathcal{Y}, \ldots$ We then provide a formulation of our intuition as follows:

(INF) for all $\mathcal{X}$, either $\mathcal{X}$ is finite or there is some $\mathcal{Y}$ witnessing that $\mathcal{X} \cong \omega$.

Intuitively, we are saying that every family of classes is finite or (simply) infinite. Thus, we deem that the cardinality of the natural numbers is the cardinality of the one and only infinite size. Since (INF) is a principle we take to be true, we incorporate it into our theory of arithmetic, classes, and families.

Now when we then come to formulate Cantor's theorem, we do not show that there is no bijection. We show, rather, that the resultant theory is inconsistent. To see this, suppose that we have a relation $\in$ in a theory of families, classes, and numbers such that

- (INF) is true; and

- some reasonable comprehension principles are in place.

Then suppose that there is a bijection $\mathcal{F}$ between naturals and all the classes of naturals. Then by our comprehension principle there is some $Y$ such that

$$
Y=\{z \mid \exists Z(\mathscr{F}(z))=Z \wedge z \notin Z\} .
$$

Then there is some $Y$ such that $\mathscr{F}(y)=Y$. Suppose that $y \in Y$. By definition, it cannot be; so $y \notin Y$. But then $y \in Y$. Thus there is no bijection $\mathcal{F}$. But this contradicts (INF) and thus our assumption that there is an $\in$ governed by the principles above is incorrect. We might call this the enumerator paradox.

Obviously, the reason why this occurs is that we have formalized our intuition about infinity and admitted it into our theory. Thus we now have a theory in which something must be wrong. Following the traditional line of attack, we reject (INF) 
and end up with the Cantorian theory of the transfinite. But we are canvassing an alternative. We contend that giving up (INF) is a significant cost and not a step to be taken lightly. Let us see what is at stake here. ${ }^{6}$

To get a sense of this, we return to our analogy with theories of truth. Tarski's theorem on the inadmissibility of truth showed us that we could not admit a predicate $T$ into our language if it was governed by the $T$-axioms. So what is the impact of this theorem? The usual answer in the truth literature is that there are (at least) a couple of ways to go here. We might say that

- the $T$-axioms do not provide the correct analysis of the concept of truth; or

- the $T$-axioms do provide the correct analysis and there is no correct formalization of truth to be had.

Recent work in the theory of truth has worked to make the distinction between these perspectives clearer (see Azzouni [1], [2], Chihara [5], Eklund [7], Patterson [25]). However, it is also possible to discern a similar thread in Tarski [31]. The first approach dictates that we have made some kind of error in our axiomatization of truth. There is a coherent concept, so to speak, out there for analysis, and Tarski's theorem shows us that this first cut at formalizing it has made a mistake. We might then look at the work carried out by Kripke [19], Tarski [32], and more recently in Field [9] as attempts to provide the correct formalization. Nonetheless, there is a sense in which these new attempts seem to miss something crucial to our conception of truth. We must either sacrifice some of the $T$-axioms or revise the logic in which these arguments are conducted. Both options present significant costs.

In contrast, the second approach acquiesces to the analysis provided by the $T$-axioms as the one which faithfully represents what it means to be true. From this perspective, Tarski's theorem then shows us that this conception is, in some sense, incoherent, or at least unformalizable. We then might look at projects undertaken by formalized truth programs such as Tarski's and Kripke's as providing, not the correct formalization of the actual concept, but rather a replacement of one which is demonstrably faulty (see Scharp [27]). We might think of a replacement theory as a felicitous approximation to our theory of truth. For example, suppose we provide a theory of truth for the language of arithmetic which only caters to those sentences which do not involve a truth predicate, that is, the arithmetic sentences. Then we would presume that any theory of truth, worthy of the name, would agree about these sentences. It is only once iterated truth contexts occur that problems arise, and even then it has to be iterated a lot. So while there may be no way of getting a perfect truth theory, we agree that there is a sense in which we can approximate it.

In parallel to the story about truth, I contend that Cantor's theorem can be understood as a theorem about inadmissibility and that this leads us to another fork in the road. Either Cantor's theorem shows us that

- the $\in$-theory including (INF) does not provide the correct analysis of infinite collections; or

- the $\in$-theory including (INF) does provide the correct analysis but there is no formal theory to be had.

Taking the first path, we end up with the traditional story. Cantor's theorem is then understood as having demonstrated that our naive theory of infinity provides a faulty analysis of our intuitive conception of infinity. We then see the set theories provided by Cantor and developed by Zermelo as attempts to provide the correct formalization 
of the concept of infinite collections. If, on the other hand, we take the second path, then we assert that our analysis of the naive conception of infinite collections is adequately represented by the theory proposed above and that Cantor's theorem shows us that no formalization of this concept is possible. We thus reject the idea that it ultimately makes sense to quantify over the collection of all classes of natural numbers. But this does not mean that we cannot take up theories which approximate that concept. Perhaps we could look at formalized set theory as a replacement of the faulty conception of the infinite with a congenial approximation to it.

In summary, we might understand the fork in the road in the following fashion. On the one hand, we get to make good sense of our talk about all the collections of natural numbers, while losing our intuitive sense of the concept of infinity. While on the other hand, taking up the solution proposed here, we retain our intuitive conception of infinity while sacrificing our ability to talk genuinely about the collection of all classes of natural numbers.

1.3.1 Should we take set theory seriously? This discussion leads us to a kind of inconsistency view about theories of infinite collections. We can only have such a theory at the cost of intuitively obvious principles. Thus, we do not take seriously the idea that there could be multiple sizes of infinity. We excommunicate the cardinals. It is a radical view, but not altogether off the books. We might think back to Boolos's question in [3] about whether we should take set theory seriously. Boolos gets us to consider some fixed points of normal functions such as the $\aleph$ and $\beth$ functions. $Z F C$ can easily deliver the existence of cardinals $\lambda$ and $\kappa$ such that $\lambda=\boldsymbol{\aleph}_{\lambda}$ and $\kappa=\beth_{\kappa}$. The argument goes through with an application of a weak form of the axiom of replacement. However, Boolos implores us to ask whether we really think there are such a staggering number of objects, so to speak, out there. In particular, he questions what it is about the axioms of $Z F C$ that we find so evident as to prompt us to accept such a prima facie outlandish conclusion. Boolos proposes that it is the iterative conception of set which provides the philosophical foundation for $Z F C$. Moreover, building upon Dana Scott's [28] work, Michael Potter [26] has further developed an axiomatic approach to the iterative conception in which the axiom of replacement has no place.

One might wonder how seriously such a challenge can be taken by the contemporary set-theoretic community which now accepts, for example, measurable cardinals as a standard part of set theory. A measurable cardinal can scarcely figure in Boolos's picture. To see this we might extend the fixed points he considers to their regular cousins. Thus, we consider the least-regular fixed points of the $\boldsymbol{\aleph}$ and $\beth$ functions: the first weak and strong inaccessible cardinals. These are much larger than the $\lambda$ and $\kappa$ that Boolos considers. But a measurable is much larger than that. If we considered any normal function (not just $\aleph$ and $\beth$ ) and demanded that they had a regular fixed point, then we would still be well below a measurable. No process from below can get anywhere near a measurable cardinal: they exhibit qualitative transcendence (see Kanamori [18]). While the reasons for the acceptance of such large cardinals are extrinsic, being related to fixing the theory of analysis, they nonetheless appear compelling to the set theorist.

Our challenge, however, probes more deeply into the core of set theory. Our concern is not whether there are cardinals of some appropriately inconceivable trans- 
finite magnitude; rather, we are worried about whether or not there are transfinite cardinalities at all. We are concerned that such talk may be ultimately incoherent.

\section{The Case of the Missing Bijection}

We observed earlier that there was something left to explain about the missing bijection given by our proof of Cantor's theorem in Section 1.1. In an effort to make a little more sense of this we make use of some tools from the philosophy of truth and set theory. We shall sketch a way of understanding the issue by using Hamkins's [15] multiverse framework while philosophically underpinning it with an adaptation of Glanzberg's [11] contextualist approach to truth.

We might describe the issue as follows. In Section 1.3, using our semiformal third-order theory, we were able to demonstrate its inconsistency in the presence of the axiom (INF), which is designed to uphold our intuitive understanding of the size of infinity. This put us in a position to reject the idea of there being a genuine notion of all classes of natural numbers over which we could quantify; thus, we leave open the question of what a good theory of classes of natural numbers should be like. However, in Section 1.1, we were able to prove a similar result on a playing field where (INF) could not be articulated. We were able to show that a certain reasonable representation of a bijection could not be present. Thus, there may be something residual left for us to explain.

We use this section to explore a way of making sense of this. We first introduce Hamkins's multiverse theory of sets and Glanzberg's contextualist theory of truth. We then combine those elements to get an idea of where the missing bijection may have, so to speak, gone.

2.1 A multiverse understanding of sets The basic idea motivating the multiverse theory of sets is that there are many different universes in which we can do set theory, none of which should be privileged over any other (see Hamkins [14], [15]). We should think of working in a different universe of sets as no different from considering different groups or rings in algebra. As such, according to the multiverse understanding, a question such as the continuum hypothesis is deflated away as a misleading way of asking in which universe we are currently. Hamkins argues for this thesis most persuasively on what we might understand as extrinsic or pragmatic grounds. He argues that mathematicians are too familiar with working in both $\mathrm{CH}$ and $\neg \mathrm{CH}$ worlds. Moreover, the fruitfulness of exploring the wide variety of settheoretic universes is also clear. As such, any argument which rejected the existence of one of those worlds should be rejected on the basis of familiarity and mathematical fecundity. It may, however, turn out that the position articulated in this paper provides a way of understanding the multiverse from a more intrinsic-and perhaps philosophically satisfying - point of view. In coming to understand that the theory of infinite collections is ultimately fraught, we hope to get a better idea of why multiple universes are natural and indeed of why such an understanding conforms to the spirit of our analysis of this troubled concept.

To get a better feeling for Hamkins's proposal, we shall look at one of the principles governing the multiverse and then consider a model of a simplified version of the system. 
For Hamkins, the multiverse of different possible set concepts is constrained by both internal and external relations. Internally, each universe is governed by familiar axioms which tell us which sets must exist. For example, the axioms of $Z F C$ would suffice for this purpose, although Hamkins would also like to consider even weaker systems such as theories of second-order arithmetic. The external relations are, however, more interesting. They tell us that if we are in one universe, then there is another universe which has an interesting relationship with the one in which we are currently. For example, Hamkins proposes that we add the following:

- Axiom of generic extensions: For every universe and any collection of approximations to an object $G$ there is a forcing extension of that universe which contains $G$.

Strictly, we probably should not call this an axiom as we have not formulated it in a first-order theory: we are using an informal language which speaks of universes and forcing. We could, of course, formalize this talk by using the language of set theory and the resources of $Z F C$, thus reducing the talk of universes to models. However, this is problematic because our multiverse should consist of genuinely alternative set-theoretic universes, not the simulacra which our traditional foundation provides: the multiverse approach is intended to provide an alternative foundation. The problem of an axiomatization of the multiverse remains open and beyond the scope of this paper; however, we remark that a synthesis of modal approaches in contemporary philosophy of set theory may provide a solution (see Hamkins and Löwe [16], Linnebo [21]).

If we put this difficult problem on hold, we can make use of our ordinary $Z F C$ foundations to give us a simpler picture of how the multiverse works. Let us then take some countable domain, say, $\omega$, and consider a system of countable transitive models of $Z F C$ closed under the axiom of generic extensions. ${ }^{7}$

Of course, in using $Z F C$ in each universe we are taking up something more powerful than the weak systems approximating second- and third-order arithmetic, which we have considered above. Moreover, it clearly omits the axiom (INF). This will allow us to keep closer in letter to Hamkins's work [15], but the important thing is that we shall still be able to see the effect of the missing bijection. We shall call such a system of models a generic multiverse (see Gitman and Hamkins [10], Woodin [34]).

2.2 Contextualism and semantic paradox We now briefly describe how a contextualist may address the liar paradox. The approach was pioneered by Parsons [24] and has been further developed and generalized to other paradoxical situations by Glanzberg [11], [12]. In a nutshell, the contextualist claims that the reason the argument for inconsistency from the liar paradox goes through is that the range of our quantifiers, and thus the salient ontology, has shifted during the argument. To see this in action, let us run through the basic argument for the contradiction by using the liar paradox and consider a simple version of the contextualist response. ${ }^{8}$ We start with the assumption that for all sentences $\varphi$ it is the case that

$$
\varphi \leftrightarrow T\ulcorner\varphi\urcorner .
$$

We then claim that there is some sentence $\lambda$ such that

$$
\lambda \leftrightarrow \neg T\ulcorner\lambda\urcorner .
$$


But $\lambda$ is itself a sentence so

$$
\lambda \leftrightarrow T\ulcorner\lambda\urcorner,
$$

and from there, we see that

$$
\lambda \leftrightarrow \neg T\ulcorner\lambda\urcorner \leftrightarrow \neg \lambda .
$$

So where have our quantifiers shifted? At the beginning of the argument, we made an assumption about how truth should work for any sentence. But which sentences count as just any sentence? The contextualist sees the inconsistency as resulting from a naive understanding of how far the quantifiers range. The simplest move for the contextualist is then something reminiscent of Tarski [32]. We say that at the beginning of the argument, when we said, "for all sentences," we only meant those sentences which did not make use of the truth predicate. On the other hand, in the next step, when we argue that there is some sentence, $\lambda$, such that $\lambda \leftrightarrow \neg T\ulcorner\lambda\urcorner$, our domain of quantification (over sentences) has shifted: we are now admitting sentences that do involve a truth predicate. Then since the domain of quantification has shifted, there is no reason to think that our first assumption applies to sentences like $\lambda$; and thus, inconsistency is averted.

Of course, this is a mere sketch which raises a couple of obvious problems. First, we might, naturally enough, think that our first assumption, or at least something like it, should apply to $\lambda$ too. This is easy enough to accommodate along Tarskian lines. We introduce a new truth predicate, say, $T_{1}$, to accommodate sentences from the new range of quantification and say of all the sentences $\varphi$ not involving $T_{1}$, that $\varphi \leftrightarrow T_{1}\ulcorner\varphi\urcorner$. We may then continue extending in this fashion, gaining a hierarchy of languages each with their own truth predicate (see Feferman [8], Halbach [13]). More seriously, we may wonder about the precise conditions under which we must shift the context of our sentential quantifiers. Glanzberg [11] has made a serious effort to address the problem by introducing a framework of propositional quantification and employing work from the contextualist literature in the philosophy of language. This is not, however, our current problem.

2.3 A contextualist approach to the multiverse via forcing We now have the tools to illustrate how the multiverse account of sets and the contextualist approach to truth can be composed together to provide an account of our missing bijection. The basic plan is as follows.

(1) Take a multiverse $M$ in which every universe $\mathcal{U}$ believes itself to contain multiple infinite cardinalities, but such that we actually see that each universe is countable.

(2) Consider the argument for Cantor's theorem as conducted in a particular universe $U$.

(3) Show how a generic extension may be understood as giving us a universe $\mathcal{U}^{\prime}$ corresponding to the context shift implicit in Cantor's theorem.

For our multiverse, we just take a generic multiverse as described above (see [34]). Given that we are following Hamkins's framework, each universe in our multiverse believes itself to contain multiple infinite cardinalities. Moreover, since we are taking $Z F C$ to be true in each of these universes, there is a sense in which the inhabitants of each universe are able to prove this by using Cantor's theorem. We now focus on how they do this, observing where a shift of context may occur. 
We take ourselves to be in a particular universe $\mathcal{U}$ in $\mathcal{M}$ and we start by taking what it thinks is the powerset of the natural numbers $\mathcal{P}(\omega)$. We then assume that there is a bijection $f: \omega \cong \mathscr{P}(\omega)$ and using separation let

$$
A=\{n \in \omega \mid n \notin f(n)\} .
$$

Since $A \in \mathscr{P}(\omega)$, there is by assumption some $a \in \omega$ such that $f(a)=A$.

But then

$$
a \in A \leftrightarrow a \notin f(a) \leftrightarrow a \notin A .
$$

As in the truth case, we claim that a shift occurs between the first and second steps. We have rejected the assumption that there is some absolute notion of the family of all classes of natural numbers, thus our move to take $\mathcal{P}(\omega)$ is merely relative to the context $U$ from which we begin the argument. But where has the context shifted to?

This is where the generic multiverse comes in. Using a forcing argument we can collapse $\mathcal{P}(\omega)$ by adding a generic object $G$ which witnesses the ultimate countability of $\mathcal{P}(\omega)$ in $\mathcal{U}$. Such an $G$ cannot be present in $\mathcal{U}$ but will be present in another universe $\mathcal{U}^{\prime}=\mathcal{U}[G]$ in $\mathcal{M}$ since it is closed under forcing extensions (see Kunen [20]). Moreover, $\mathcal{U}[G]$ will be the minimal extension of $\mathcal{U}$ which still upholds the axioms of $Z F C$. So we make sense of the missing bijection as a generic object $G$. It is not present in the initial context $\mathcal{U}$ but there is always another context $\mathcal{U}^{\prime}$ in which it is.

Observing this situation and given our claim that there are not any really uncountable infinities, we might imagine ourselves as, so to speak, navigating an endless collection of these countable models in something like the generic multiverse we have described. While the illusion of multiple infinite cardinalities is witnessed inside each of the universes, we are free to move between them. ${ }^{9}$

But all this should cause us to ask the questions: Why should we accept the forcing extension axiom? What is so natural about it?

In an effort to provide a more philosophical response, I would like to make the provocative suggestion that forcing is a kind of natural revenge or dual to Cantor's theorem: where Cantor gives us the transfinite, forcing tears it down. But what is interesting about this suggestion? Why would we think there is any natural relationship between the two approaches? In defense of this claim, I would like to gesture at an obvious similarity between the way we prove Cantor's theorem and the way we show that generic objects cannot exist in our universe.

To illustrate this, we first provide a quick précis of the forcing technique. In brief, we start with a ground model $\mathcal{M}$ and construct, so to speak, a generic object $G$ from a series of approximations to it. Each of these approximations is in $\mathcal{M}$ and indeed, we can collect together all the different ways of constructing such a $G$ in a partial-order $\mathbb{P}$ which is also an element of $\mathcal{M}$. We then consider particular subsets $D$ of the partial order $\mathbb{P}$ which we call dense. Intuitively speaking, a dense set $D$ contains a set of approximations such that no matter which way you tried to assemble a $G$ using $\mathbb{P}$ there would always be another approximation $p$ in $D$ to which you could extend your current approximation. A set $G$ is generic if it ends up using some of every dense $D \subseteq \mathbb{P}$.

Our generic extension principle then tells us that there is a universe in which such a $G$ must occur, but we also want to know that $G$ was not already in $\mathcal{M}$. The argument for this follows that of Cantor in a step-by-step fashion. We illustrate this in Table 1. 
Table 1

\begin{tabular}{|l|l|}
\hline Cantor & Cohen \\
\hline Suppose the existence of a surjection & Suppose the existence of a \\
$f: \omega \rightarrow \mathcal{P}(\omega)$. & $G: \omega^{\mathcal{M}} \rightarrow \lambda^{\mathcal{M}} \in \mathcal{M}$. \\
\hline Then consider the set $A=\{n \in \omega \mid n \notin f(n)$. & Then consider the set $E=\{p \in \mathbb{P} \mid p \notin G\}$, \\
& which is dense. \\
\hline Observe that since $f$ is surjective $\exists a$ such that & Observe that since $E$ is dense $\exists e \in E$ such that \\
$f(a)=A$. & $e \in G$. \\
\hline We then have $a \in A \leftrightarrow a \notin f(a) \leftrightarrow a \notin A$. & We then have $e \in E \leftrightarrow e \notin G \leftrightarrow e \notin E$. \\
\hline Thus, there is no such $f$. & Thus, $G \notin M$. \\
\hline
\end{tabular}

The tentative lesson I would like to draw from this is that Cantor's theorem and Cohen's are two sides of the same coin. Rather than seeing forcing and generic extensions as mere, albeit powerful, instruments in the set theorist's toolkit, I am proposing that we see forcing as a deep insight that results from our analysis of the theory of infinite collections. We should perhaps see the axiom of generic extensions as exactly the kind of thing which our experience with infinite collection should force upon us: it is intrinsic.

2.3.1 Why not Skolem hulls? We observe that a more traditional approach in this vicinity is a species of Skolem relativism. In contrast to the approach proposed in this paper, this kind of Skolem relativist approach uses the downward LöwenheimSkolem theorems (rather than Cohen's) to show that any first-order theory with a reasonable axiomatization has a countable model. From this, we are encouraged to draw skeptical conclusions about the viability of multiple infinite cardinalities (see von Neumann [33]). Despite the similarities in the final positions of these approaches, we have opted against the Skolem approach here. Our main reason is that, in using the downward Löwenheim-Skolem theorem, we have to start, so to speak, in a model which is uncountable before we apply it. So there is a sense in which we start off in the very place whose ultimate coherence we already doubt. This seems less congenial than the generic multiverse approach in that there we may start with countable models and move between them by transforming those models: the move is additive rather than subtractive. Moreover, since our starting position is always countable, we minimize our indulgence of the transfinite realm.

2.3.2 A remark about indefinite extensibility As a final remark for this section, we observe that there is a species of indefinite extensibility at work in this sketch. Dummett [6] describes the notion of indefinite extensibility as follows:

An indefinitely extensible concept is one such that, if we can form a definite conception of a totality all of whose members fall under that concept, we can, by reference to that totality, characterize a larger totality all of whose members fall under it [6, p. 441].

The archetypal example of such a concept is Russell's concept of a set which is not a member of itself. If we take a definite totality of sets which are not members of themselves, then we may then characterize a new, larger totality. Shapiro and Wright [29] have attempted to make sense of this by demanding that a definite conception of a totality be a set. This results in a notion of indefinite extensibility where a concept is indefinitely extensible if it can be put in correspondence with the ordinals: indefinite extensibility is reduced away, so to speak, to the prior concept of the absolute infinity of the ordinals. 
In contrast to this kind of formulation, the indefinite extensibility witnessed above is horizontal, so to speak, rather than vertical. Given a particular universe, we claim that there will always be another one extending it to a wider one which contains witnesses to its ultimate countability. The forcing construction allows us to take an existing totality (our current universe) and characterize a larger totality which contains all the elements of the original totality.

Dummett also appears to have recognized something very close to what we are suggesting in this paper:

The argument [for Cantor's theorem] does not show that the real numbers form a non-denumerable totality unless we assume at the outset that they form a determinate totality comprising all that we shall ever recognise as a real number: the alternative is to regard the concept real number as an indefinitely extensible one [6, p. 442].

Our contextualist sketch takes up the position that there is no determinate totality comprising all that we shall ever recognize as a real number. Moreover, we take it that the argument from (INF) to inconsistency demonstrates that there is no such totality to be had. Rather, we are restricted to particular contexts or universes. As such, like Dummett, we treat the concept of a real number as indefinitely extensible.

\section{Problems}

So that is the view we are entertaining. We now examine some of the obvious problems for it.

(1) Is there a naive conception of infinity?

(2) Sociological differences between theories of truth and theories of infinity.

(3) Where do we stop?

(4) What about simpler problems of cardinality and the transfinite?

3.1 Is there a naive conception of infinity? The intelligibility of the view proposed crucially depends on there being a naive conception of infinity which does not permit more than one size of infinity. Without this, the analogy between infinity and truth fails. However, a conceivable point of difference between accounts of truth and of infinite collections is the work done by intuitiveness. It seems plausible (and has certainly been defended) that the $T$-axioms constitute in the meaning of truth. If you were asked the question: what does "true" mean, we could explain it saying that we are "entitled to assert (or deny) a sentence that it is true precisely under the circumstances when we can assert (or deny) the sentence itself" (see [19]). This is, loosely speaking, just an informal characterization of the $T$-axioms. Moreover, while the analysis is shrewd, it is also very intuitive. It appears to accord well with our usual use of the term and as such, it seems quite reasonable to attribute to the average person such a naive conception of truth.

On the other hand, we might think differently about the concept of infinity. At face value, it is a mathematical concept and as such, the characterization of its meaning should be left to the experts: mathematicians. For example, we would not want to trust the meaning of the term "natural number" to the man on the street. Characterizing such a concept is surely the task of a mathematician or philosopher of mathematics. With this particular example, one reason for this is that the concept of natural numbers is difficult to characterize. A reasonable characterization of the laws of arithmetic requires a recursive axiomatization and, as such, seems well beyond the 
abilities of the layperson. On the other hand, the concept of infinity is easy enough to grasp, despite its vertiginous quality. Infinity is that quantity beyond which there is no larger. What exactly that means may end up depending a lot on the context in which it is articulated. However, in the case of arithmetic and its theory of families and classes, it seems relatively straightforward. A family is infinite if every other family is either smaller than it or has the same size. ${ }^{10}$ The definition is not particularly complicated or subtle. On this basis, I would like to contend that there is, indeed, a sense in which we possess a naive conception of infinity.

3.2 Historical differences between theories of truth and theories of infinity Another potential weakness in the analogy emerges in the history of theoretical approaches to these problems. The study of theories of truth is extremely diverse. There are a plethora of different theories and logics of truth, each defended for the most part in plausible ways. This kind of diversity lends a certain credence to the idea that truth is ultimately problematic. On the other hand, the theory of the transfinite is relatively unified. Moreover, once the axiom of choice has been admitted, the world of the transfinite is very well behaved. This unification could lend credence to the claim that the transfinite is not problematic. I do not have much to say on what effect this distinction should have on the analogy proposed. However, if this were the driving reason why there is less plurality in theories of infinity, then that would seem interesting in its own right.

3.3 Where do we stop? Another problem for the view proposed above is the question of its scope. An important feature of the analogy above is the dependence of both liar reasoning and Cantorian reasoning on a diagonal argument. We might be tempted to take this as implying that there is something fishy going on in diagonal arguments, and we should perhaps alleviate dependence upon them in our core theories. But of course this will not do. It would be difficult to enumerate the value of diagonal arguments in mathematical logic. To take just a small selection, without them we would lose Gödel's theorem, Turing's halting problem, the Baire category theorem, and the Borel hierarchy theorem. We do not want to throw the baby out with the bathwater.

So what is stopping us from taking other diagonal arguments and saying that they too are paradoxical like the liar? I think the answer is that we can, but to make the analogy work some argument still needs to be made to show that an intuitive principle has been violated.

Let us consider the halting problem in more detail. To take up an inconsistentist approach to recursion theory, we need to argue that some intuitive principle has been violated. But what would this be? Let us quickly review the argument.

Theorem 3.1 There is no total recursive function $f: \omega \rightarrow \omega$ which verifies whether or not an arbitrary partial recursive function halts on its own code number.

Proof Suppose not. Then there is a total recursive function $f: \omega \rightarrow \omega$ such that for any algorithm code $e \in \omega$, we have

$$
f(e)= \begin{cases}1 & \text { if } \varphi_{e}(e) \text { halts } \\ 0 & \text { otherwise }\end{cases}
$$


Given this, we then construct the partial recursive function $\varphi_{k}: \omega \rightarrow \omega$ such that

$$
\varphi_{k}(n)= \begin{cases}\infty & \text { if } f(n)=1, \\ 0 & \text { otherwise. }\end{cases}
$$

Now supposing that $\varphi_{k}$ is coded by $k$, let use consider what happens when we present $k$ to $\varphi_{k}$. We get

$$
\varphi_{k}(k) \text { halts } \leftrightarrow \varphi_{k}(k)=0 \leftrightarrow f(k)=0 \leftrightarrow \varphi_{k}(k) \text { does not halt, }
$$

which is a contradiction, thus there is no such recursive function $f$.

What intuitive principle has been violated in this argument? In analogy with our Cantorian rejection, we might say that our efforts to quantify over all of the partial recursive function were flawed in the first place. Thus our total recursive function is bound to miss something, in analogy with the missing bijection.

But an important difference creeps in here. In the cardinality case, we had the idea that we could not quantify over all the subsets of the naturals and so the important bijection was necessarily missing. The bijection was, however, understood to become available in some larger context. But even this new bijection is relative: it only works for the classes of the previous context. This allows us to retain our intuition that nothing is "really" uncountable, merely relatively so.

However, in the case of the halting problem, our goal is to establish the existence of, so to speak, an absolute function capable of verifying all of the facts about partial recursive functions, regardless of context. The argument above establishes that this cannot be done in the local context, and a fortiori we shall fail to find such an absolute function when we move across multiple contexts too.

3.4 What about simpler problems of cardinality and the transfinite? Throughout this narrative, I have unquestioningly presupposed the idea that cardinality should be understood via bijection. However, the naive conception of cardinality also seems to involve something like subset-hood. If we have a finite collection of objects and consider a proper subset of that collection, then the subset is smaller in cardinality than the original collection. It is only when we consider infinite collections that this property can fail. So given that we are questioning Cantor's conception of the transfinite, perhaps we should also be questioning the use of bijection as well. If I am arguing for one intuitive notion of cardinality, should I not be defending this one too?

I think there are a couple of ways for me to respond here. First, we could acknowledge that there are further nuances in the theory of cardinality which involve the move from the finite to the infinite. A serious attempt at providing a nonbijective equinumerosity account can be found in the second half of Mancosu's work [23]. The essential problem is to find a natural total ordering on infinite collections of natural numbers which extends the partial order on the underlying powerset algebra. This is a fascinating project, but it is outside the scope of this paper. Moreover, it has little effect on its claims. Even if such an project could be successful, the problems for the bijective story still stand. Perhaps the conclusions of this paper could be used to lend philosophical support to such projects. We might also make the stronger claim that in finite contexts, strict subset-hood implies that "less than" is merely symptomatic rather than characteristic of the cardinality concept. In finite cases, it is merely sufficient but not necessary: there are finite collections which have less cardinality than 
others but are not subsets of them. Thus we claim that it would be better to restrict our attention to the content which is definitive of cardinality.

\section{Conclusion}

Our goal in this paper has been to elucidate an alternative philosophical interpretation of Cantor's theorem. Rather than understanding it as foundation for a highway into the transfinite, we have attempted to understand it as speaking about the ultimate problems of quantifying over infinite collections and the difficulties in forming coherent theories thereof. To bolster our case, we have drawn heavily on an analogy with theories of truth and the problem of the liar paradox. In the second section, we have taken this analogy a step further and made an opening move toward a contextualist diagnosis of the problem, making use of recent research into multiverse theories of sets. Finally, we have attempted to address some of the more obvious objections and issues for the position proposed here.

\section{Notes}

1. By way of background, I first entertained this idea when considering an issue that emerged in a response by Florio and Shapiro to a paper by Linnebo and Rayo [22]. The issue in question concerned what occurs when we make the move from an interpreted language to its metatheory. It seemed obvious that something like the powerset of the original domain was required to think about its metatheory in a natural way. But after further consideration, this now seems less obvious.

2. It is worth noting that a much stronger (indeed impredicative) comprehension principle is required to generate Russell's paradox. In neo-Fregean projects, this leaves open the possibility of revising comprehension to get out of trouble (see Burgess [4]).

3. Further discussion of the relationship between the position proposed in this paper and intuitionism is discussed in Section 2.3.2.

4. $\ulcorner\varphi\urcorner$ is a natural number coding the formula $\varphi$.

5. Mancosu lays out the four main positions historically taken with regard to infinite collections:

(1) Use the paradoxical features that such infinities would have (one is a part of another, i.e., one infinity is smaller than another) to declare this strictly impossible and thus block the process of generation of such infinities ...

(2) Accept infinite collections but deny that 'greater than', 'less than', and 'equality' can be applied to infinities ...

(3) Accept infinities and analyze how different part-whole relations apply to infinities than those that apply to finite quantities ...

(4) Accept infinities and try to develop an arithmetic of the infinite in analogy to the arithmetic of the finite [23, p. 616].

6. We should note here the relationship between our argument here and what is usually known as Cantor's paradox. This is usually presented in the form of a theorem:

Theorem There is no largest cardinal. 
Proof Assume the contrary, and let $C$ be the largest cardinal number. Then (in the von Neumann formulation of cardinality) $C$ is a set and therefore has a powerset $2^{C}$ which, by Cantor's theorem, has cardinality strictly larger than that of $C$. Demonstrating a cardinality (namely that of $2^{C}$ ) larger than $C$, which was assumed to be the greatest cardinal number, falsifies the definition of $C$. This contradiction establishes that such a cardinal cannot exist.

We prove this theorem with the tools of set theory. However, it is easy enough to adapt the argument into our framework. It is essentially just the argument we have provided, but rather than assuming (INF), the argument is used to establish that (INF) is incorrect.

7. We assume well-foundedness to make the models relatively well behaved. However, we could also consider multiverses in which this condition is jettisoned (see [15]).

8. Glanzberg [11] makes use of a framework admitting propositional quantification, which we shall not need here. He claims that a richer semantic framework admitting propositions is required to make what is actually happening in the context shifts clear. However, as our target is somewhat different, we may avoid that extra complexity here.

9. This discussion might lead us to suspect that a kind of revenge phenomenon is in the offing. Reasonably enough, we might be led to ask how this theory of countable models works. But if we respond to this by providing a first-order theory that talks about these countable models, then it will probably be able to formulate something akin to Cantor's theorem; and thus, we will end up saying something like there are uncountably many countable models. This is an interesting problem, but a full response is outside the scope of this paper. We do, however, provide an outline of three main lines of response.

(1) We might say that the story of multiple universes is pretheoretic and not susceptible to formalization. This means that talk of functions whose domains and ranges are models is unavailable and Cantor's theorem is blocked. This is the simplest response, but also the least philosophically satisfying. The request to provide some (of course, incomplete) axiomatization of the theory of the multiverse seems reasonable.

(2) On the other hand, we might allow the theory of multiple universes to be formalized, and further, concede that some form of Cantor's theorem is likely to be provable. Thus it appears that we have established that there are uncountably many objects. However, we might then respond that the multiverse principles should still apply to this newly formalized system. So just as we allowed different universes of our set theory in the generic multiverse, we should allow different multiverses of our multiverse theory. Thus, we shift to a second-order multiverse (of multiverses) in which, for example, we would demand that for any multiverse in the second-order multiverse another multiverse is present which witnesses its countability. We then claim that since the second-order multiverses are countable, there are not really uncountably many countable models in any of them.

(3) Finally, we might design a formal system which is capable of axiomatizing the multiverse and which accommodates the response of (2) but does not lead into a theoretical regress. Ideally, such a system would be able to characterize both the internal and external principles of the multiverse in such a way that the external multiverse principles are also upheld as internal principles. A modal approach or something altogether novel may be appropriate here.

10. Now of course, there is the further question of what it means for one collection to be larger than another. Let us take it that we have already gained acceptance of the equinumerosity of the naturals and the even numbers. Thus we take it the lack of a surjection 
from class $X$ to class $Y$ would mean that $Y$ is larger than $X$. There are other theories of cardinality which do not use bijection (see [4]). See Section 3.4.

\section{References}

[1] Azzouni J.,"The strengthened liar, the expressive strength of natural languages, and regimentation," Philosophical Forum, vol. 34 (2003), pp. 329-50. 198

[2] Azzouni, J., "The inconsistency of natural languages: How we live with it," Inquiry, vol. 50 (2007), pp. 590-605. 198

[3] Boolos, G., "Must we believe in set theory?," pp. 120-32 in Logic, Logic, and Logic, edited by R. Jeffrey, Harvard University Press, Cambridge, Mass., 1998. MR 1675856. 199

[4] Burgess, J. P., Fixing Frege, Princeton University Press, Princeton, 2005. Zbl 1089.03001. MR 2157847. 208, 210

[5] Chihara, C., "The semantic paradoxes: A diagnostic investigation," Philosophical Review, vol. 88 (1979), pp. 590-618. 198

[6] Dummett, M., "What is mathematics about?," pp. 11-26 in Mathematics and Mind (Amherst, Mass, 1991), Logic and Computation in Philosophy, Oxford University Press, New York, 1994. MR 1373891. 204, 205

[7] Eklund, M., "Inconsistent languages," Philosophy and Phenomenological Research, vol. 64 (2002), pp. 251-75. 198

[8] Feferman, S., "Reflecting on incompleteness," Journal of Symbolic Logic, vol. 56 (1991), pp. 1-49. Zbl 0746.03046. MR 1131728. DOI 10.2307/2274902. 202

[9] Field, H., Saving Truth from Paradox, Oxford University Press, Oxford, 2008. MR 2723032. DOI 10.1093/acprof:oso/9780199230747.001.0001. 198

[10] Gitman, V., and J. D. Hamkins, "A natural model of the multiverse axioms," Notre Dame Journal of Formal Logic, vol. 51 (2010), pp. 475-84. MR 2741838. DOI 10.1215/00294527-2010-030. 201

[11] Glanzberg, M., "The liar in context," Philosophical Studies, vol. 103 (2001), pp. 217-51. MR 1838987. DOI 10.1023/A:1010314719817. 200, 201, 202, 209

[12] Glanzberg, M., "Context and unrestricted quantification," pp. 45-74 in Absolute Generality, edited by A. Rayo and G. Uzquiano, Oxford University Press, Oxford, 2006. 201

[13] Halbach, V., “Tarski hierarchies,” Erkenntnis, vol. 43 (1995), pp. 339-67. MR 1396842. DOI 10.1007/BF01135378. 202

[14] Hamkins, J. D., "Some second order set theory," pp. 36-50 in Logic and Its Applications, edited by R. Ramanujan and S. Sarukkai, vol. 5378 of Lecture Notes in Computer Science, Springer, Berlin, 2009. MR 2540935. DOI 10.1007/978-3-540-92701-3_3. 200

[15] Hamkins, J. D., "The set-theoretic multiverse," Review of Symbolic Logic, vol. 5 (2012), pp. 416-49. MR 2970696. DOI 10.1017/S1755020311000359. 200, 201, 209

[16] Hamkins, J. D., and R. Löwe, “The modal logic of forcing," Transactions of the American Mathematical Society, vol. 360 (2008), pp. 1793-1817. Zbl 1139.03039. MR 2366963. DOI 10.1090/S0002-9947-07-04297-3. 201

[17] Hodges, W., "An editor recalls some hopeless papers," Bulletin of Symbolic Logic, vol. 4 (1998), pp. 1-16. Zbl 0979.03002. MR 1609195. DOI 10.2307/421003. 192

[18] Kanamori, A., The Higher Infinite: Large Cardinals in Set Theory from Their Beginnings, Springer Monographs in Mathemathics, Springer, Berlin, 2003. MR 1994835. 199

[19] Kripke, S., "Outline of a theory of truth," Journal of Philosophy, vol. 72 (1975), pp. 690-716. 198, 205 
[20] Kunen, K., Set Theory: An Introduction to Independence Proofs, Elsevier, Sydney, 2006. 203

[21] Linnebo, Ø., "Pluralities and sets,” Journal of Philosophy, vol. 107 (2010), pp. 144-64. 201

[22] Linnebo, Ø., and A. Rayo, "Hierarchies ontological and ideological," Mind, vol. 121, no 482 (2012), pp. 269-308. DOI 10.1093/mind/fzs050. 208

[23] Mancosu, P., "Measuring the size of infinite collections of natural numbers: Was Cantor's theory of infinite number inevitable?" Review of Symbolic Logic, vol. 2 (2009), pp. 612-46. MR 2583924. DOI 10.1017/S1755020309990128. 196, 207, 208

[24] Parsons, C., Mathematics in Philosophy: Selected Essays, Cornell University Press, Ithaca, N.Y., 1983. MR 0728948. 201

[25] Patterson, D. E., "Tarski, the liar and inconsistent languages," The Monist, vol. 89 (2006), pp. 150-77. 198

[26] Potter, M. D., Set Theory and Its Philosophy: A Critical Introduction, Oxford University Press, New York, 2004. Zbl 1066.03002. MR 2050123. DOI 10.1093/acprof:oso/9780199269730.001.0001. 199

[27] Scharp, K., "Alethic vengeance," in Revenge of the Liar: New Essays on the Paradox, edited by J. C. Beal, Oxford University Press, Oxford, 2007. 198

[28] Scott, D., "Axiomatizing set theory," pp. 207-14 in Proceedings of Symposia in Pure Mathematics, vol. 13, part 2, American Mathematical Society, Providence, 1974. 199

[29] Shapiro, S., and C. Wright, "All things indefinitely extensible," pp. 255-304 in Absolute Generality, edited by A. Rayo and G. Uzquiano, Oxford University Press, Oxford, 2006. 204

[30] Simpson, S. G., Subsystems of Second Order Arithmetic, Springer, Berlin, 1999. MR 1723993. DOI 10.1007/978-3-642-59971-2. 193, 194

[31] Tarski, A., " The semantic conception of truth and the foundations of semantics," Philosophy and Phenomenological Research, vol. 4 (1944), pp. 341-76. MR 0010521. 195, 198

[32] Tarski, A., "The concept of truth in formalized languages," in Logic, Semantics, Metamathematics: Papers from 1923 to 1938, edited by J. Corcoran, Clarendon Press, Oxford, 1956. MR 0078296. 195, 198, 202

[33] von Neumann, J., "An axiomatization of set theory," in From Frege to Gödel: A Source Book in Mathematical Logic, 1879-1931, edited by J. van Heijenoort, Harvard University Press, Cambridge, Mass., 1967. Zbl 51.0163.04. 191, 204

[34] Woodin, W. H., "The realm of the infinite," pp. 89-118 in Infinity: New Research Frontiers, edited by M. Heller and W. H. Woodin, Cambridge University Press, Cambridge, 2011. Zbl 1283.03082. MR 2767235. DOI 10.1017/CBO9780511976889.006. 201, 202

\section{Acknowledgments}

I would like to thank Matti Eklund, Michael Glanzberg, Patrick Greenough, Graham Priest, Tim Button, Colin Caret, Sean Walsh, and Zach Weber for many useful conversations about this subject matter. I thank the Plurals, Predicates and Paradox project and the Foundations of Logical Consequence project for giving me the opportunity to present these ideas. Finally, I would like to thank an anonymous referee whose comments and suggestions have made this paper much better. 
Department of Philosophy University of Aberdeen

50-52 College Bounds

Aberdeen AB24 3DS

UK

toby.meadows@gmail.com 